\title{
Operação de Senning com a utilização de tecidos do próprio paciente
}

\author{
Luiz Fernando CANÊO*, Domingos D. LOURENÇO FILHO*, Roberto ROCHA E SILVA*, Sonia M. \\ FRANCHI ${ }^{*}$, Jorge Y. AFIUNE*, Cristina M. Camargo AFIUNE*, Amilcar O. MOCELIN*, \\ Miguel BARBERO-MARCIAL ${ }^{\star *}$, Fabio B. JATENE**
}

RBCCV 44205-471

\begin{abstract}
Canêo L F, Lourenço Filho D D, Rocha e Silva R, Franchi S M, Afiune J Y, Afiune C M C, Mocelin A O, Barbero-
Marcial M, Jatene F B - Operação de Senning com a utilização de tecidos do próprio paciente. Rev Bras Cir Cardiovasc 1999; 14 (4): 298-302.
\end{abstract}

\begin{abstract}
RESUMO: O grande atrativo da Operação de Senning como descrita originalmente é a utilização das paredes do próprio átrio do paciente para a realização dos túneis venosos. A atrioseptostomia por balão, utilizada atualmente na grande maioria dos casos de transposição das grandes artérias (TGA), cria grandes comunicações interatriais. Com isso, é necessário a utilização de enxertos biológicos ou sintéticos (Dacron, Teflon e pericárdio bovino) na septação do átrio esquerdo. Doze crianças consecutivas, com idades de 5 meses a 4 anos (média = 20 meses), foram submetidas à Operação de Senning com a utilização de tecidos do próprio paciente. O diagnóstico era de TGA em 9, TGA com comunicação interventricular (CIV) em 1, TGA com estenose pulmonar valvar discreta em 1, TGA com justaposição das aurículas em 1 caso e todas as crianças foram submetidas à atrioseptostomia com balão no período neonatal. Em 5 casos foi utilizada a aurícula esquerda aberta e invertida para a septação interatrial, 1 com inversão e, em 5 , inversão com abertura da aurícula esquerda e uso do próprio pericárdio in situ para a realização do túnel das veias pulmonares, devido às reduzidas dimensões do átrio direito ( 1 caso de justaposição das aurículas). O tempo de internação variou de 10 a 24 dias (média 15 dias), sendo que o período de pós-operatório variou de 7 a 22 dias (média 12 dias). Não houve óbitos no período de internação hospitalar. O seguimento no pós-operatório foi de 8 a 34 meses (média 23 meses) e todos os pacientes apresentaram boa evolução clínica. O ecocardiograma realizado no período de internação e no seguimento pós-operatório não evidenciou sinais de obstrução ao fluxo nos túneis intracardíacos. O uso de tecidos do próprio paciente in situ, com potencial possibilidade de crescimento, resgata a vantagem principal da técnica originalmente descrita por Senning.
\end{abstract}

DESCRITORES: Transposição dos grandes vasos, cirurgia. Procedimentos cirúrgicos cardíacos, métodos. Retalhos cirúrgicos.

\section{INTRODUÇÃO}

A técnica descrita inicialmente por SENNING (1) para a correção da transposição das grandes artérias (TGA) no plano atrial, através da criação de túneis intracardíacos era realizada apenas com retalhos do próprio tecido atrial, pela possibilidade de crescimento durante o desenvolvimento da criança.
Com o aparecimento da correção anatômica pela Operação de Jatene ${ }^{(2)}$, a operação no plano arterial passou a ser a técnica de escolha para a correção da TGA, apresentando os melhores resultados a longo prazo, quando realizada no período neonatal.

A correção no plano atrial passou, então, a ser empregada em crianças maiores, muitas vezes submetidas previamente a procedimentos de amplia-

\footnotetext{
Trabalho realizado no Instituto do Coração do Hospital das Clínicas da Faculdade de Medicina da Universidade de São Paulo e no Hospital do SEPACO (Projeto InCor - SEPACO). São Paulo, SP, Brasil.

* Do Hospital do SEPACO.

** Do Instituto do Coração do Hospital das Clínicas da Faculdade de Medicina da Universidade de São Paulo.

Recebido para publicação em agosto de 1999.

Endereço para correspondência: Luiz Fernando Canêo. Av. Dr. Enéas de Carvalho Aguiar, 44. Divisão Cirúrgica. São Paulo, SP, Brasil. CEP: 05403-000. Tel: (011) 3069-5432.e-mail: caneo@ibm.net
} 
ção da comunicação interatrial, seja através da septostomia por balão (RASHKIND \& MILLER (3)) ou pela atrioseptectomia pela técnica de BlalockHanlon (4). Nesses casos, a realização da Operação de Senning na presença de grandes comunicações interatriais torna necessária a utilização de enxertos biológicos ou sintéticos (Dacron, Teflon e pericárdio bovino) na septação do átrio esquerdo, acima das veias pulmonares.

No grupo de crianças onde encontramos átrio direito (AD) de pequenas dimensões, situação observada nos casos de justaposição das aurículas, a falta de tecido pode comprometer a anastomose das veias pulmonares direitas com o remanescente da parede do $A D$, dificultando a drenagem venosa pulmonar. Nesses casos a Operação de Senning só é possível com a utilização de retalhos para ampliação do AD.

Várias opções técnicas ${ }^{(5-9)}$ foram descritas para evitar o emprego de enxertos biológicos ou sintéticos nessas situações. Dentre elas, podemos citar a sutura da aurícula esquerda invertida aberta ou não na septação dos átrios e a utilização de pericárdio do paciente in situ no redirecionamento das veias pulmonares para a valva tricúspide.

Este estudo mostra a nossa experiência com a utilização dessas modificações técnicas, que permitiram a realização da Operação de Senning com a utilização de tecidos do próprio paciente.

\section{CASUÍSTICA E MÉTODOS}

No período de junho de 1995 a março de 1998, 12 crianças, com idades de 5 meses a 4 anos (média = 20 meses), foram consecutivamente submetidas à Operação de Senning na Unidade Cirúrgica do SEPACO - Instituto do Coração do Hospital das Clínicas da Faculdade de Medicina da Universidade de São Paulo.

Os diagnósticos foram: TGA em 9, TGA com comunicação interventricular (CIV) em 1, TGA com estenose pulmonar valvar discreta em 1, TGA com justaposição das aurículas em 1 caso. Todas as crianças foram submetidas à atrioseptostomia com balão no período neonatal e apresentaram algum tipo de intercorrência que impossibilitou a realização da correção anatômica na época adequada.

\section{Técnica Cirúrgica}

Através da esternotomia mediana convencional, faz-se a canulação da aorta ascendente e das veias cavas, estas com o emprego de cânulas venosas aramadas de ponta metálica (Medtronic DLP - Grand
Rapids, MI - USA). Sob hipotermia moderada em $28^{\circ} \mathrm{C}$ e cardioplegia sangüínea fria, é realizada a atriotomia direita, alguns centímetros abaixo da crista terminalis, como classicamente descrito.

Após inspeção da anatomia intracavitária, realiza-se a incisão no sulco interatrial, acima das veias pulmonares direitas. Isso permite maior mobilidade do septo interatrial remanescente. Realiza-se, então, a inversão da aurícula esquerda, observandose a possibilidade de fechamento completo do septo interatrial acima das veias pulmonares. Caso a aurícula se mostre insuficiente para a septação, a mesma é aberta com uma incisão em "T" em sua parede anterior, longitudinalmente do ápice à base $\mathrm{e}$, depois, estendida transversalmente, paralelo à junção apêndice-atrial, como descrita por ASSAD et al. (5). Esse retalho é, então, suturado de forma contínua com polipropilene 6-0, observando-se os detalhes técnicos bem estabelecidos para a criação do assoalho do átrio venoso sistêmico.

Nos casos de anatomia favorável, o teto do seio coronariano é aberto (10) para que a drenagem do sangue seja redirecionada para a valva mitral, juntamente com o retorno venoso das cavas.

As cavas são, então, redirecionadas para a valva mitral através do túnel realizado com as paredes do átrio direito, suturado abaixo da valva tricúspide, com sutura contínua de prolene 6-0. Posteriormente, executa-se a anastomose das veias pulmonares com o remanescente do átrio direito para completar a técnica descrita por SENNING (1) Nos casos em que encontramos tecido atrial insuficiente para a realização do túnel, foi utilizado pericárdio do paciente in situ. Esse retalho é facilmente obtido através de duas incisões verticais, uma junto ao diafragma até a cava inferior e outra ao nível da cava superior. Obtem-se, assim, um retalho quadrangular, com sua base inferior junto às veias pulmonares direitas, em continuidade com a parte posterior do pericárdio. O nervo frênico é cuidadosamente separado do pericárdio, de maneira que seja possível suturar a parede atrial esquerda com o retalho, logo acima da chegada das veias pulmonares. Os bordos superior e inferior são suturados sobre a parede lateral da cava superior e inferior, o bordo anterior ao remanescente da parede atrial, completando, assim, o túnel das veias pulmonares com a valva tricúspide.

\section{RESULTADOS}

Em todos os casos foi realizada a Operação de Senning apenas com a utilização de tecidos do próprio paciente. Em 5 casos foi utilizada a aurícula esquerda aberta e invertida para a septação 
Canêo L F, Lourenço Filho D D, Rocha e Silva R, Franchi S M, Afiune J Y, Afiune C M C, Mocelin A O, Barbero-Marcial M, Jatene F B Operação de Senning com a utilização de tecidos do próprio paciente. Rev Bras Cir Cardiovasc 1999; 14 (4): 298-302.

interatrial, sendo em apenas 1 caso a sua inversão e, em 5, inversão com abertura da aurícula esquerda e uso de pericárdio do paciente in situ para a realização do túnel das veias pulmonares, devido ao pequeno tamanho do átrio direito. Destes, somente 1 caso apresentava justaposição de aurículas.

O tempo de circulação extracorpórea variou de 115 a 180 min (média 142 min) e o tempo médio de isquemia miocárdica variou de 90 a 130 min (média $108 \mathrm{~min}$ ). O tempo de internação variou de 10 a 24 dias (média 15 dias), sendo que o tempo de pósoperatório variou de 7 a 22 dias (média 12 dias).

Não houve óbitos no período de internação hospitalar.

O seguimento variou de 8 a 34 meses (média 23 meses) e todos os pacientes apresentaram boa evolução clínica.

O ecocardiograma realizado no período de internação e no seguimento pós-operatório não evidenciou sinais de obstrução ao fluxo nos túneis intracardíacos.

\section{COMENTÁRIOS}

O tratamento cirúrgico da transposição das grandes artérias pode ser dividido em três grandes períodos. No período inicial, realizava-se somente operações paliativas. Posteriormente, apareceram as operações de correção no plano atrial ou ventricular e, mais recentemente, a correção anatômica, que marca o momento atual.

Entre as técnicas de correção no plano atrial, podemos citar a Operação de Senning (1) e a de Mustard (11).

A técnica descrita inicialmente por SENNING (1) tinha como objetivo a criação de túneis intracardíacos, com a inversão do fluxo sistêmico e pulmonar na chegada dos ventrículos. Era realizada apenas com retalhos do próprio tecido atrial da criança.

Mais tarde, MUSTARD (11) descreve uma técnica, onde os desvios intracardíacos são realizados com a utilização de um retalho único de pericárdio do paciente ou outro tecido sintético ou biológico de mais fácil execução, passando a ter grande aceitação por diversos grupos cirúrgicos.

Posteriormente, QUAEGEBEUR et al. (12) reviveram a técnica de Senning, apresentando algumas modificações técnicas e reforçando a importância da utilização de retalhos das paredes atriais apenas na criação dos túneis intracardíacos com a potencial possibilidade de crescimento.
Em 1975, JATENE et al. (2) realizaram a primeira correção anatômica com sucesso, em um paciente com TGA e CIV. Embora inicialmente descrita para esse grupo de pacientes, e indicada para crianças maiores, com o ventrículo esquerdo ainda adaptado para a circulação sistêmica, alguns grupos mostraram a sua grande aplicabilidade em recém-nascidos, com excelentes resultados, incluindo os casos de TGA com septo interventricular íntegro $(13,14)$.

As operações no plano atrial, como a Operação de Senning, foram colocadas em segundo plano e indicadas apenas para os casos que perderam a oportunidade de indicação da Operação de Jatene.

Em nosso meio, a correção anatômica da TGA ainda apresenta mortalidade hospitalar relativamente maior que a experiência mundial (15). É possível que estas diferenças relacionem-se ao grande número de crianças que se apresentam tardiamente aos nossos Centros de Cardiologia Pediátrica, já com ventrículos esquerdos desadaptados, além das dificuldades inerentes no manuseio do recém-nascido. Isso torna-se evidente quando analisamos a distribuição etária das crianças encaminhadas para o tratamento cirúrgico da TGA em nossa Instituição. Embora observemos um aumento no número de operações em crianças com idade inferior a 30 dias, somente em torno de $50 \%$ é realizada a Operação de Jatene (apresentado por Jatene, AD, em: $2^{\text {nd }}$ International Symposium of Pediatric Cardiology: Brazilian Experience - São Paulo, SP, novembro de 1997).

As anomalias de coronária, quando presentes na TGA, dificultam a realização da correção anatômica (10) e deverão ser consideradas na seleção dos pacientes, principalmente pelos grupos de menor experiência cirúrgica com esse tipo de máformação, bem como o baixo peso ao nascimento. Nesses casos, devemos lembrar que a Operação de Senning continua sendo uma ótima opção técnica, podendo ser realizada com baixa mortalidade, mesmo em Serviços não altamente especializados.

O grande atrativo da Operação de Senning sobre a de Mustard é a utilização de tecidos do próprio paciente ${ }^{(12)}$. Porém, nas crianças maiores, submetidas previamente à atrioseptostomia com balão, a criação de grandes comunicações interatrias dificulta a septação dos átrios sem a utilização de um retalho biológico ou sintético.

No intuito de evitar a utilização desses enxertos, algumas modificações técnicas foram descritas (5-9), como a inversão da aurícula que permite, em alguns casos, a septação do átrio esquerdo acima das veias pulmonares. Nas crianças onde ela se apresenta pequena e insuficiente para essa septação, a sua inversão e abertura em $T$ invertido na base permite a criação de um grande retalho atrial, cerca de três vezes maior que o obtido apenas com a sua inver- 
Canêo L F, Lourenço Filho D D, Rocha e Silva R, Franchi S M, Afiune J Y, Afiune C M C, Mocelin A O, Barbero-Marcial M, Jatene F B Operação de Senning com a utilização de tecidos do próprio paciente. Rev Bras Cir Cardiovasc 1999;14 (4):298-302.

são. Devemos salientar o cuidado a ser tomado com essa abertura, observando que a incisão não se estenda muito além da sua base, com riscos de lesão da artéria circunflexa. Nesse local pode ocorrer, ainda, sangramento significativo e grande atenção deve ser dada à sutura nessa região, que deve ser realizada com muito cuidado, utilizando fios finos (prolene 6-0 ou 7-0). Uma vez que a revisão cirúrgica desse local é de difícil acesso, devemos lembrar que é obrigatório a sua realização antes da saída de CEC, onde eventuais sangramentos devem ser eficientemente corrigidos. Nos casos de quantidade insuficiente de tecido atrial $D$ para a tunelização das veias pulmonares para o ventrículo direito (VD), principalmente observada nos casos de justaposição das aurículas, onde dispomos de poucas opções técnicas (16), podemos utilizar o pericárdio do paciente in situ. Esse retalho é facilmente obtido pela sua secção junto a veia cava superior e de sua inserção diafragmática, observando-se a proximidade do nervo frênico, e a manutenção da irrigação do retalho. O pericárdio do paciente in situ suturado ao AD permite a tunelização das veias pulmonares para o VD sem grandes dificuldades, com a possibilidade potencial de crescimento durante o desenvolvimento da criança.

O resultado a longo prazo das correções de TGA no plano atrial está relacionado às obstruções dos túneis intracardíacos, às arritmias atriais e ao desempenho do VD submetido às pressões sistêmicas $(10,17)$. Se observamos que apenas $10 \%$ dos pacientes apresentam disfunção ventricular severa, e a sobrevida de $80 \%$ dos casos em 20 anos (10), podemos considerar a importância dessa técnica no tratamento da TGA nas crianças que perderam a oportunidade da realização da Operação de Jatene.

As arritmias estão presentes em torno de 50\% ou mais dos casos em 10 anos ${ }^{(18)}$ e não observamos diferença evidente entre as técnicas de Mustard e Senning $(19,20)$. Pouco podemos fazer para diminuir sua incidência, pois elas estão relacionadas às suturas interatriais necessárias como parte da técnica.

A obstrução dos túneis intracardíacos parece estar relacionada diretamente ao tipo de enxerto utilizado, como observado de forma mais evidente nas crianças submetidas à operação de Mustard, e nos casos onde retalhos sintéticos são empregados (21).

Com a utilização de tecidos com potencial possibilidade de crescimento, como descrito na nossa experiência, podemos, teoricamente, minimizar essa complicação tardia; no entanto, maior tempo de seguimento se faz necessário.

RBCCV 44205-471

Canêo L F, Lourenço Filho D D, Rocha e Silva R, Franchi S M, Afiune J Y, Afiune C M C, Mocelin A O, BarberoMarcial M, Jatene F B - Senning operation with autologous tissue for atrial septum augmentation or pulmonary venous pathway enlargement. Rev Bras Cir Cardiovasc 1999;14 (4): 298-302.

ABSTRACT: One of the main theoretical advantages of the original Senning Operation is the ability to avoid either biologic or synthetic patches for atrial septum augmentation or to enlarge pulmonary venous pathways. To accomplish this we have performed the Senning procedure with some modifications that allow the operation to be completed without the use of heterologous tissue. Twelve children, aged 5 months to 4 years (mean age: 20 months) with transposition of great arteries who had previous balloon atrial septostomy were submitted to the Senning procedure. The following associated lesions were diagnosed: ventricular septal defect in 1 patient, valvular pulmonary stenosis in 1 and auricular juxtaposition in 1 . The modified technique used for atrial septum augmentation was the use of open inverted left auricula in 5 cases, inverted auricula in 2 and in situ autologous pericardium for venous pathway enlargement in 5 . The hospitalization was 10 to 24 days (mean $=15$ days) and the post-operative period was 7 to 22 days (mean $=12$ days). There were no in-hospital or late deaths during a mean follow-up period of 23 months (range 8 to 34 months). All patients had a good clinical outcome and their follow-up echocardiographic examinations showed no evidence of venous pathway obstruction. This modified Senning Operation can be performed without either biologic or synthetic patches for atrial septum augmentation or to enlarge pulmonary venous pathway. The use of autologous in situ tissues with potential for growth, retains the main theoretical advantage of the original technique.

DESCRIPTORS: Transposition of great vessels, surgery. Cardiac surgery procedures, methods. Surgical flaps. 


\section{REFERÊNCIAS BIBLIOGRÁFICAS}

1 Senning A - Surgical correction of transposition of the great vessels. Surgery 1959; 45: 966-80.

Jatene A D, Fontes V F, Paulista P P et al. - Successful anatomic correction of transposition of the great vessels: a preliminary report. Arq Bras Cardiol 1975; 28: $461-4$

3 Rashkind W J \& Miller W W - Creation of an atrial septal defect without thoracotomy: a palliative approach to complete transposition of the great arteries. JAMA 1966; 196: 991-2.

4 Hanlon C \& Blalock A - Complete transposition of the aorta and pulmonary artery: experimental observations on venous shunts as corrective procedures. Ann Surg 1948; 127: 385-97.

5 Assad R S, Felipe H S, Marcial M B, Jatene A D - The Senning operation: a new technique to prepare the septal flap. Ann Thorac Surg 1991; 51: 678-9.

6 Eufrate S \& Luisi V - A further modification of the Senning operation. Thorac Cardiovasc Surg 1981; 29: 88-90.

7 Hamilton J, Wilson N, Dickinson D, Walker D - A modification of the Senning procedure for complete transposition. In: Crupi G, Parenzan L, Walker R, eds. Perspectives in pediatric cardiology. Mount Kisco, NY: Futura, 1981: 48-51.

8 Levinsky L, Srinivasan V, Alvarez-Dias F, Subramanian $S$ - Reconstruction of the new atrial septum in the Senning operation: new technique. $J$ Thorac Cardiovasc Surg 1981; 81: 131-4.

9 Ingram M T, Von Segesser L, Ott D A, Huhta J C, Murphy D J Jr. - Senning repair for transposition of the great arteries without patch augmentation of the septum. J Thorac Cardiovasc Surg 1988; 96: 485-7.

10 Kirklin J \& Barratt-Boyes B - Complete transposition of great arteries. In: Cardiac Surgery. 2nd ed. White Plains, New York: Churchil Livingstone, 1993: $1383-467$.

11 Mustard W - Successful two-stage correction of transposition of the great vessels. Surgery $1964 ; \mathbf{5 5}$ 469-72.

12 Quaegebeur J M, Rohmer J, Brom A G - Revival of the
Senning operation in the treatment of transposition of the great arteries: preliminary report on recent experience. Thorax 1977; 32: 517-24.

13 Castaneda A R, Norwood W I, Jonas R A, Colon S D, Sanders $S$ P, Lang $P$ - Transposition of the great arteries and intact ventricular septum: anatomical repair in the neonate. Ann Thorac Surg 1984; 38: 438-43.

14 Castaneda A - Arterial switch operation for simple and complex TGA: indication criterias and limitations relevant to surgery. Thorac Cardiovasc Surg 1991; 39 (Suppl 2): 151-4.

15 Souza L \& Jatene A D - Correção anatômica da transposição das grandes artérias. Rev Soc Cardiol do Estado de São Paulo 1992; 2: 62-72.

16 Barbero-Marcial M, Verginelli G, Vila J, Zerbimi E J Transposition of the great arteries (TGA) associate with total anomalous pulmonary venous connection (TAPVC): a surgical approach. Ann Thorac Surg 1984; 37: 92-4.

17 DeLeon V H, Hougen T J, Norwood W I, Lang P, Marx G R, Castaneda A - Results of the Senning operation for transposition of the great arteries with intact ventricular septum in neonates. Circulation 1984; 70 (3 Pt 2): 121-5

18 Williams W G, Trusler G A, Kirklin J W et al. - Early and late results of a protocol for simple transposition leading to an atrial switch (Mustard) repair. $J$ Thorac Cardiovasc Surg 1988; 95: 717-26.

19 Marx G R, Hougen T J, Norwood W I, Fyler D C Castaneda A R, Nadas A S - Transposition of the great arteries with intact ventricular septum: results of Mustard and Senning operations in 123 consecutive patients. J Am Coll Cardiol 1983; 1 (2 Pt 1): 476-83.

20 Martin T C, Smith L, Hernandez A, Weldon C S Dysrhythmias following the Senning operation for dextro-transposition of the great arteries. J Thorac Cardiovasc Surg 1983; 85: 928-32.

21 Paul M H \& Wernovsky G - Transposition of the great arteries. In: Emmanouilides G C, Riemenschneider T A, Allen H D, Gutgesell H T, eds. Moss and Adams' Heart disease in infants, children, and adolescents: including the fetus and young adult. 5 ed. Baltimore, Williams \& Wilkins, 1995: 1154-224 